\title{
Kyphoplasty for Malignant Mesothelioma Metastasis to the L5 Vertebra for Spine Stabilization
}

\author{
David Walega ${ }^{1}$ and Benjamin Liu ${ }^{2}$ \\ ${ }^{1}$ Department of Anesthesiology, Feinberg School of Medicine, Northwestern University, 251 East Huron Street, F5-704, Chicago, IL 60611, USA \\ ${ }^{2}$ Department of Radiology, Feinberg School of Medicine, Northwestern University, 251 East Huron Street, Chicago, IL 60611, USA \\ Address correspondence to David Walega,d-walega@northwestern.edu
}

Received 11 June 2012; Accepted 23 April 2013

Copyright ( 92013 David Walega and Benjamin Liu. This is an open access article distributed under the terms of the Creative Commons Attribution License, which permits unrestricted use, distribution, and reproduction in any medium, provided the original work is properly cited.

\begin{abstract}
Malignant mesothelioma (MM) is an aggressive primary malignancy which invades the pleura, peritoneum or pericardium. Mortality rates are high. Local tumor invasion is inevitable; and metastasis to the chest wall, mediastinum, or thoracic spine is common. Distal metastasis to osseous structures other than the thoracic spine is rare. External beam radiation therapy (EBRT) is a mainstay treatment for metastatic disease in the spine but carries significant risks of myelopathy and unintended damage to normal surrounding tissues and is insufficient when mechanical instability of the affected vertebra is present. Further, EBRT may take up to 2 weeks to elicit meaningful pain relief. Balloon kyphoplasty (KP) is a brief, safe, outpatient, image-guided percutaneous procedure performed with either twilight sedation or general anesthesia. The ideal KP candidate has midline non-radiating pain which worsens with weight bearing and direct palpation at the site of vertebral metastasis or fracture, and pain which improves in the supine position. We present a rare case of L5 vertebral metastasis from end-stage recalcitrant MM treated with KP for pain control and vertebral body stabilization. KP should be considered as a palliative treatment option, with strong evidence to suggest predictable pain relief and improved function when appropriate candidates are selected for the procedure. Caregivers should be aware of the indications of KP as well as expected outcomes.
\end{abstract}

Keywords malignant mesothelioma; vertebral metastasis; vertebral augmentation; palliative care; cancer pain management; kyphoplasty; vertebral stabilization

\section{Introduction}

Malignant mesothelioma (MM) is an aggressive primary malignancy which invades the pleura, peritoneum, or pericardium. Mortality rates are high. Local tumor invasion is inevitable; and metastasis to the chest wall, mediastinum, or thoracic spine is common. Distal metastasis to other osseous structures is rare. We present a unique case of L5 vertebral treated with balloon kyphoplasty (KP) for vertebral body stabilization and pain control.

\section{Case report}

A 51-year-old Hispanic male presented with a 2-month history of non-radiating central low back pain and a five year history of MM. At the time of his initial diagnosis with MM, a complete right extrapleural pneumonectomy with diaphragm and pericardial resection and lymph node biopsy was performed. At that time, he was classified with Stage III T2 N1 M0 epithelioid mesothelioma. Following surgery, he underwent four cycles of cisplatin and pemetrexed chemotherapy followed by radiation. Three years later, when new pulmonary lesions were identified, an additional six cycles of chemotherapy was administered. One year later, liver metastasis was identified and treated with five cycles of gemcitabine with palliative radiation using $50 \mathrm{~Gy}$ followed by two cycles of vinorelbine.

At the time of his presentation with low back pain complaints, the patient reported a numeric rating scale (NRS) for pain of $5 / 10$ when it was most severe. NRS typically varied from 5-7/10. He was using 50-100 oral morphine equivalents per day with partial improvement in pain symptoms and moderate side effects. His physical examination was remarkable for tenderness with palpation and percussion over the L5 spinous process and the paralumbar muscles. His neurologic exam was normal except for decreased light touch in a stocking distribution to the ankles bilaterally, consistent with chemotherapyinduced neuropathy. Computed tomography (CT) of the lumbar spine confirmed two soft tissue metastatic lesions in the right posterior paraspinal muscles and a lesion within the L5 vertebral body. Magnetic resonance imaging (MRI) confirmed a T2 intermediate signal, STIR hyperintense enhancing lesion involving the majority of the anterior L5 vertebra with fractures of the superior and inferior endplates and $60 \%$ height loss (Figure 1). Incidental grade I-II anterolisthesis of L5 on S1 was seen with chronic pars defects bilaterally at this level.

Given the patient's advanced disease, low pulmonary reserves, and failure of adjuvant therapy to arrest metastasis, 


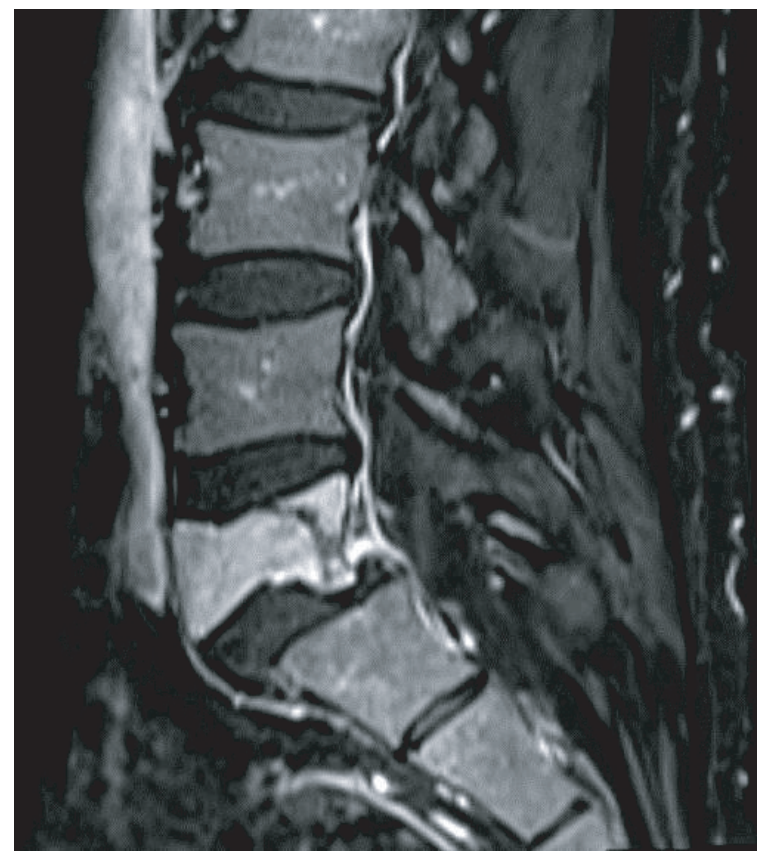

Figure 1: Gadolinium enhanced MRI of the lumbar spine shows replacement of the anterior L5 vertebral body by an avidly enhancing mass, consistent with metastatic disease. The posterior L5 vertebral body and bilateral pedicles were preserved without metastasis.

a lumbar stabilization surgery was not recommended. As an alternative, balloon kyphoplasty (KP) was offered to stabilize the L5 vertebra, halt further collapse at this level, and decrease pain symptoms. KP was performed at L5 with biplanar fluoroscopic guidance with polymethylmethacrylate (PMMA). While most of the cement was injected into the lytic cavity, a small volume of cement was also injected into the posterior vertebral body and bilateral pedicles, bridging the lytic cavity and trabecular bone in order to improve stability in both the anterior and posterior spinal columns (Figure 2). Core biopsies taken during the KP procedure showed cells strongly positive for keratin AE1/AE3, consistent with epithelioid mesothelioma. A post-kyphoplasty CT scan showed a reduction in vertebral collapse (Figure 3). Following the procedure the patient reported 50\% reduction in low back pain and he remained ambulatory. Two weeks later, he abruptly succumbed to a pulmonary infection and ventilatory failure unrelated to the procedure.

\section{Discussion}

$\mathrm{MM}$ is an aggressive primary malignancy of the serosal membranes: the pleura, peritoneum, and pericardium. It is related to exposure to mineral fibers such as asbestos and erionite, but is also seen in the absence of environmental triggers. Epithelioid mesothelioma is the most common

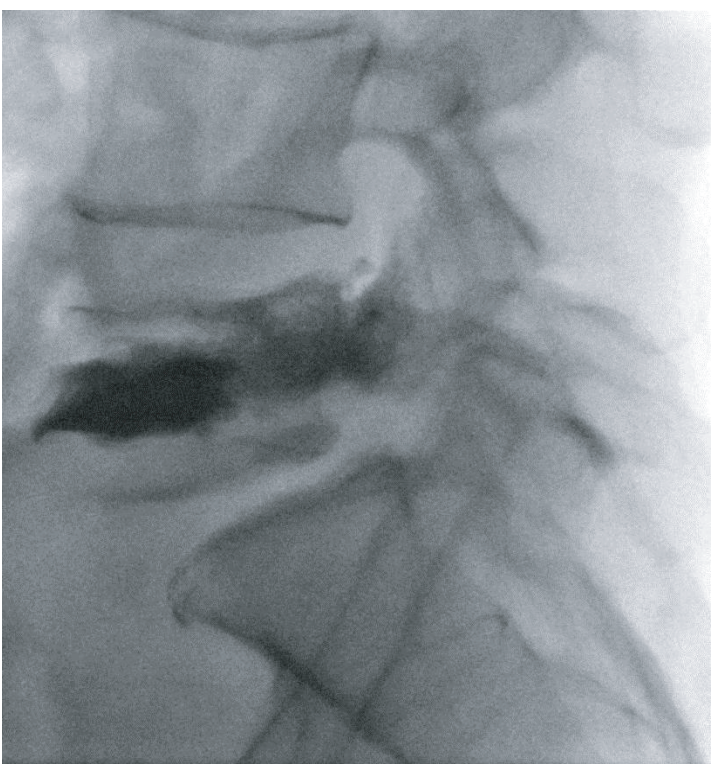

Figure 2: Lateral view fluoroscopic image after KP demonstrates PMMA fill of the lytic cavity in the anterior L5 vertebral body bridged to trabecular bony PMMA fill in the posterior L5 vertebral body and bilateral pedicles. Trace, clinically inconsequential extravasation of PMMA cement into a small paraspinal vein is noted in the left inferior L4/5 neuroforamen.

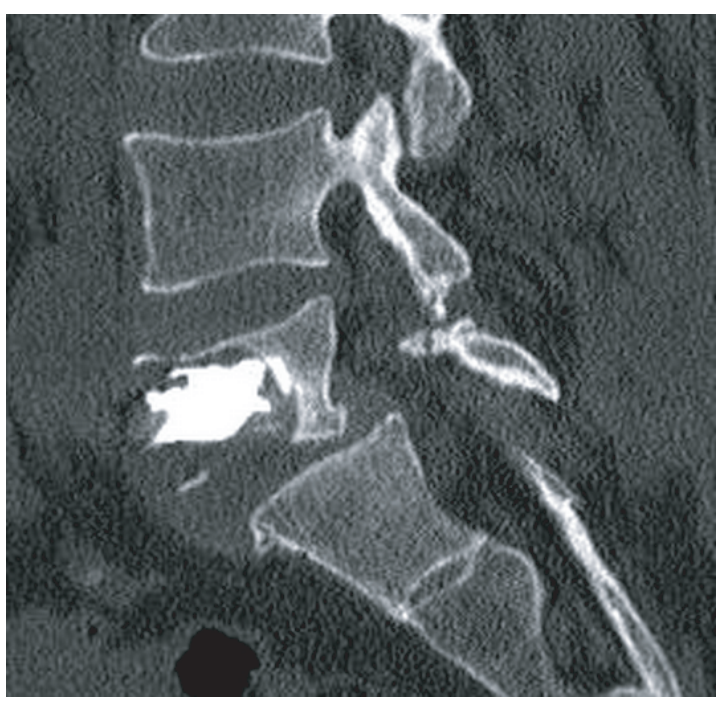

Figure 3: Post-KP CT demonstrates PMMA filling the majority of the lytic cavity.

subtype, but sarcomatoid and mixed histologies can also be seen [5]. Epidemiologic data suggests a multifactorial etiology of MM with an inter-play of mineral fiber exposure, genetic predisposition, viral infection, or exposure to ionizing radiation $[5,12]$. The incidence of $\mathrm{MM}$ in the U.S. ranges from 1 to 15 cases/million and varies 
regionally. In the United States alone, it is responsible for 3,000 deaths per year [17]. Following diagnosis, median survival is 14 months in those who undergo surgical resection [10] and less than 1 year in those with nonresectable disease [3]. Pleural mesothelioma, the most prevalent, is more common in men; while primary peritoneal disease has less gender predisposition. Women with MM are less likely to have exposure to asbestos or erionite as compared to men. As many other malignancies mimic the histopathology of mesothelioma, the diagnosis is confirmed with immunochemistry markers $[4,5,13]$.

Patients initially present with pulmonary symptoms or abdominal complaints depending on the location of the primary lesion. Pleural effusions are common. Local recurrence occurs in $80 \%$ of cases following surgical resection [29], and metastatic disease is noted in 10$50 \%$ of MM cases, more common at late stages of the disease [19]. Tumor invasion may be related to direct invasion of surrounding tissues at the site of a primary lesion in addition to hematogenous or lymphatic spread. MM commonly metastasizes to the chest wall, mediastinum and thoracic spine; but rare cases of metastasis to the oral cavity [22], face [6], medullary spinal cord [7], stomach [14], testicles [1], mandible [27], humerus [18], and femur [19] have been published.

As yet, there is no universally accepted treatment approach to MM. The optimal timing and type of surgery, chemotherapy or radiation are not established. Cisplatin and pemetrexed combination chemotherapy is a common treatment based on the clinical efficacy observed in $41 \%$ of subjects in a phase III clinical trial that showed a small increase in mean survival [28]. Repeat chemotherapy treatment may be efficacious in some cases [15].

KP was introduced in 2001 as a treatment for vertebral body fractures. The procedure aims to reduce subjective pain and stabilize the involved vertebrae and in some cases can correct kyphotic deformity. It is a brief percutaneous procedure that can be performed with either sedation or general anesthesia on an outpatient basis. The ideal KP candidate has midline non-radiating pain which worsens with weight bearing and direct palpation at the site of the fracture [16].

Pain is the most common presenting symptom of spine metastasis. Neurologic signs may be seen with retropulsion of bone fragments or compression of the spinal cord, but such signs rarely exceed axial pain [9]. Breast, prostate, lung, renal, and hematopoeitic tumors commonly metastasize to the thoraco-lumbar spine, as a large volume of bone marrow is present for potential tumor cell deposition [26]. Overall, the incidence of spinal metastasis is $60 \%$ in the thoracic region, $30 \%$ in the lumbar region, and $10 \%$ in the cervical region [8].

In patients with pathologic vertebral body compression, $\mathrm{KP}$ and external beam radiation therapy (EBRT) are often used together. EBRT is a mainstay treatment of metastatic disease in the spine, but carries a risk of myelopathy [25] and unintended damage to normal surrounding tissues. EBRT alone does not suffice when obvious mechanical instability of the vertebra is present and tumor cell death may cause further collapse and instability. Though pain relief is seen in $75 \%$ of patients treated solely with EBRT, it may take up to 2 weeks for patients to notice this effect, depending on the radiosensitivity of the tumor [20]. In contrast, pain relief following KP is seen within 24 hours and is expected in $>70 \%$ of patients [29]. PMMA has cytotoxic and thermolytic effects on tumor cells which may result in immediate tumor cell death. Cement within the cancelous bone occludes feeder vessels to the tumor, decreasing available tumor nutrition [9]. Stereotactic radiosurgery can also be effective for instability and pain [25].

Only a few well designed studies of outcomes in KP for metastatic spine lesions have been published. In a retrospective study of 48 patients with 124 metastatic spine lesions who underwent KP, Qian et al. reported a decrease in VAS from $7.4 \pm 2.1$ pre-operatively to $3.8 \pm 1.6$ following KP $(P<.001)$. Oswestry scores of disability improved by $50 \%$ and social functioning improved as well. The etiology of vertebral metastasis in these study patients was breast or lung cancer, but only 2/124 lesions treated with KP were at the L5 level [23]. None of these patients had MM.

In another retrospective study, Hirsch et al. analyzed 201 cases of metastatic spine lesions treated with either KP or vertebroplasty, an alternative method of vertebral augmentation. The most common primary neoplasms were multiple myeloma, breast cancer or lung cancer. They found 39\% of patients treated with vertebral augmentation experienced complete relief of pain after the procedure, regardless of the technique used. There was no correlation between type of cancer and the clinical outcome of pain relief. No analysis of vertebral level treated and pain outcome following the procedure was done. None of these cases involved MM and very few KPs were done at the L5 level [16].

In another comprehensive review of 12 published studies of KP performed for malignant spinal lesions, which included over 300 patients, universally improved pain scores and functional outcomes were seen [21].

The cancer patient fracture evaluation study (CAFE) is the first published prospective, randomized trial of KP in cancer patients with symptomatic spine metastases [2]. The study included 134 subjects with cancer and spine metastases who were randomized to undergo either $\operatorname{KP}(n=70)$ or non-surgical management ( $n=64)$, and were then evaluated on a variety of outcome measures: Roland-Morris Questionnaire, SF36, and pain scores. Pain scores decreased by $50 \%$ in the KP group at 1 week; and statistically and clinically significant improvements in disability, function, quality of life, and pain scores were seen for the 12-month duration of 
the study in this treatment group consistent with retrospective data previously summarized. Similar to most of these studies, however, spinal lesions were most commonly from breast and lung cancers and were located in the thoracic or upper lumbar spine. None of these subjects had MM and very few of the lesions were in the L5 vertebra.

The extent and location of osseous tumor invasion is a significant factor in assessing candidacy for KP. Lesions involving more than $35 \%$ of the vertebral body or invading the pedicles increase the risk of vertebral collapse [24]. KP is contraindicated in asymptomatic vertebral lesions without instability, severe cord compression from retropulsed bone fragments with myelopathy, severe spinal stenosis, uncorrectable coagulopathy, local or systemic infection, and allergy to the KP components like PMMA. Disruption of the posterior vertebral body cortex will require greater vigilance during $\mathrm{KP}$, but is not an absolute contraindication. Greater than $75 \%$ vertebral body compression makes KP technically difficult or impossible and typically no more than three levels are treated at one time [11]. Surgical decompression may be necessary if radiculopathy from bone fragments or tumor is identified [8,23].

\section{Conclusion}

To our knowledge this is the first report of L5 vertebral body metastasis from MM treated with KP. In this case, tumor invasion into the posterior vertebra had seriously compromised structural integrity of the spinal segment, putting the patient at high risk of complete vertebral collapse. KP addressed the patient's pain related to bony tumor involvement and avoided a more invasive spinal stabilization surgery, relatively contraindicated due to the patient's low pulmonary reserves and high tumor load. Residual back pain following the procedure was likely related to ongoing progression of soft tissue metastasis in the paralumbar muscles. These outcomes concur with recently published prospective and retrospective studies.

In cases of vertebral metastasis wherein pain or vertebral instability is noted, KP should be considered as a palliative treatment option, with strong evidence to suggest predictable pain relief and improved function when appropriate candidates are selected for the procedure. Hospitalists and palliative care teams should be aware of the indications of $\mathrm{KP}$ as well as expected outcomes in these patients.

\section{References}

[1] K. Abe, N. Kato, K. Miki, S. Nimura, M. Suzuki, H. Kiyota, et al., Malignant mesothelioma of testicular tunica vaginalis, Int J Urol, 9 (2002), 602-603.

[2] J. Berenson, R. Pflugmacher, P. Jarzem, J. Zonder, K. Schechtman, and J. B. Tillman, Balloon kyphoplasty versus non-surgical fracture management for treatment of painful vertebral body compression fractures in patients with cancer: a multicentre, randomised controlled trial, Lancet Oncol, 12 (2011), 225-235.
[3] P. Bertino, F. Piccardi, C. Porta, R. Favoni, M. Cilli, L. Mutti, et al., Imatinib mesylate enhances therapeutic effects of gemcitabine in human malignant mesothelioma xenografts, Clin Cancer Res, 14 (2008), 541-548.

[4] M. Carbone, R. A. Kratzke, and J. R. Testa, The pathogenesis of mesothelioma, Semin Oncol, 29 (2002), 2-17.

[5] M. Carbone, B. H. Ly, R. F. Dodson, I. Pagano, P. T. Morris, U. A. Dogan, et al., Malignant mesothelioma: facts, myths, and hypotheses, J Cell Physiol, 227 (2012), 44-58.

[6] D. S. Cassarino, W. Xue, and K. J. Shannon, Widespread cutaneous and perioral metastases of mesothelioma, J Cutan Pathol, 30 (2003), 582-585.

[7] M. C. Chamberlain, K. D. Eaton, J. R. Fink, and T. Tredway, Intradural intramedullary spinal cord metastasis due to mesothelioma, J Neurooncol, 97 (2010), 133-136.

[8] S. Dalbayrak, M. R. Onen, M. Yilmaz, and S. Naderi, Clinical and radiographic results of balloon kyphoplasty for treatment of vertebral body metastases and multiple myelomas, J Clin Neurosci, 17 (2010), 219-224.

[9] H. Deramond, C. Depriester, P. Toussaint, and P. Galibert, Percutaneous vertebroplasty, Semin Musculoskelet Radiol, 1 (1997), 285-296.

[10] R. M. Flores, T. Routledge, V. E. Seshan, J. Dycoco, M. Zakowski, Y. Hirth, et al., The impact of lymph node station on survival in 348 patients with surgically resected malignant pleural mesothelioma: implications for revision of the American Joint Committee on Cancer staging system, J Thorac Cardiovasc Surg, 136 (2008), 605-610.

[11] D. R. Fourney, Vertebroplasty versus kyphoplasty in the cancer setting: rethinking the relative indications, Support Cancer Ther, 3 (2005), 26-27.

[12] J. E. Goodman, M. A. Nascarella, and P. A. Valberg, Ionizing radiation: a risk factor for mesothelioma, Cancer Causes Control, 20 (2009), 1237-1254.

[13] F. Gueugnon, S. Leclercq, C. Blanquart, C. Sagan, L. Cellerin, M. Padieu, et al., Identification of novel markers for the diagnosis of malignant pleural mesothelioma, Am J Pathol, 178 (2011), 1033-1042.

[14] H. Hayashi, K. Notohara, H. Yoshioka, T. Matsuoka, H. Ikeda, $\mathrm{K}$. Kagawa, et al., Localized malignant pleural mesothelioma showing a thoracic mass and metastasizing to the stomach, Intern Med, 49 (2010), 671-675.

[15] H. Hayashi, I. Okamoto, Y. Ichikawa, M. Miyazaki, H. Yoshioka, $\mathrm{K}$. Kunimasa, et al., Retreatment of recurrent malignant pleural mesothelioma with cisplatin and pemetrexed, Int J Clin Oncol, 15 (2010), 497-499.

[16] A. E. Hirsch, R. M. Jha, A. J. Yoo, A. Saxena, A. Ozonoff, M. J. Growney, et al., The use of vertebral augmentation and external beam radiation therapy in the multimodal management of malignant vertebral compression fractures, Pain Physician, 14 (2011), 447-458.

[17] R. Ismail-Khan, L. A. Robinson, C. C. Williams Jr., C. R. Garrett, G. Bepler, and G. R. Simon, Malignant pleural mesothelioma: a comprehensive review, Cancer Control, 13 (2006), 255-263.

[18] R. N. Laurini, Diffuse pleural mesothelioma with distant bone metastasis. A case report, Acta Pathol Microbiol Scand A, 82 (1974), 296-298.

[19] T. Lester and H. Xu, Malignant pleural mesothelioma with osseous metastases and pathologic fracture of femoral neck, Appl Immunohistochem Mol Morphol, 16 (2008), 507-509.

[20] Y. Lievens, K. Kesteloot, A. Rijnders, G. Kutcher, and W. Van den Bogaert, Differences in palliative radiotherapy for bone metastases within Western European countries, Radiother Oncol, 56 (2000), 297-303.

[21] E. Mendel, E. Bourekas, P. Gerszten, and J. D. Golan, Percutaneous techniques in the treatment of spine tumors: what are 
the diagnostic and therapeutic indications and outcomes?, Spine (Phila Pa 1976), 34 (2009), S93-S100.

[22] S. Moser, M. Beer, G. Damerau, H. T. Lübbers, K. W. Grätz, and A. L. Kruse, A case report of metastasis of malignant mesothelioma to the oral gingiva, Head Neck Oncol, 3 (2011), 21.

[23] Z. Qian, Z. Sun, H. Yang, Y. Gu, K. Chen, and G. Wu, Kyphoplasty for the treatment of malignant vertebral compression fractures caused by metastases, J Clin Neurosci, 18 (2011), 763 767.

[24] P. S. Rose and J. M. Buchowski, Metastatic disease in the thoracic and lumbar spine: evaluation and management, $\mathrm{J}$ Am Acad Orthop Surg, 19 (2011), 37-48.

[25] D. M. Sciubba, T. Nguyen, and Z. L. Gokaslan, Solitary vertebral metastasis, Orthop Clin North Am, 40 (2009), 145-154.

[26] H. Taneichi, K. Kaneda, N. Takeda, K. Abumi, and S. Satoh, Risk factors and probability of vertebral body collapse in metastases of the thoracic and lumbar spine, Spine (Phila Pa 1976), 22 (1997), 239-245.

[27] N. Terakado, S. Shintani, K. Nakashiro, and H. Hamakawa, Malignant pleural mesothelioma metastasis to the mandible, Int J Oral Maxillofac Surg, 33 (2004), 798-800.

[28] N. J. Vogelzang, J. J. Rusthoven, J. Symanowski, C. Denham, E. Kaukel, P. Ruffie, et al., Phase III study of pemetrexed in combination with cisplatin versus cisplatin alone in patients with malignant pleural mesothelioma, J Clin Oncol, 21 (2003), 26362644.

[29] M. G. Zauderer and L. M. Krug, The evolution of multimodality therapy for malignant pleural mesothelioma, Curr Treat Options Oncol, 12 (2011), 163-172. 